\title{
La casa di chi? Da territorialità esclusive verso territorialità inclusive: rileggere il lavoro di comunità in contesti di nuova immigrazione
}

\author{
Paolo Boccagni, Silvia Volpato*
}

\begin{abstract}
Whose home? From exclusionary to inclusionary territories. Community work in new immigration contexts

This article revisits immigrant newcomers' local settlement, by looking at their homemaking practices, their local identifications and their everyday interactions with natives and long-term residents. By analyzing some community development initiatives in Trento, we critically discuss the prospects and dilemmas of social cohesion policies in new immigrant receiving communities. As our case study shows, local settlement is affected by preexisting social infrastructures and by the orientation of public policy, but also by two more critical factors: on the one hand, the possibility to understand and mediate between different views of home; on the other, the interplay between different ways of framing, attending and using neghbourhood spaces as more or less "home-like".
\end{abstract}

Keywords: immigration, territory, local integration, associations, homemaking, community work

\section{Introduzione}

La dimensione locale, per chi guarda ai territori come palcoscenico ma anche come "co-produttori" dell'esperienza di vita quotidiana, è un ambito privilegiato di analisi dei processi di insediamento e di inclusione/esclusione sociale delle persone immigrate. In questo contributo ne rivisitiamo le funzioni e le potenzialità a partire da una forma di territorialità minimale, quotidiana ed essenziale: la casa. Questa si presta a essere intesa non solo come luogo materiale di residenza, ma anche come spazio simbolico, identitario ed emotivo, che non sempre coincide con il domicilio; che può essere alimentato da specifici vissuti emotivi (il sentirsi a casa in un

Saggio Proposto alla Redazione il 30-5-2018 accettato il 29-9-2018

" Università di Trento, ERC HOMInG, paolo.boccagni@unitn.it; Associazione trentina accoglienza stranieri, silvia.volpato@atas.tn.it.

Silvia Volpato, operatrice di comunità di ATAS onlus, ha partecipato alla realizzazione dei progetti TRA.Mi.Te e INTEREST e si occupa di sviluppo e lavoro di comunità nell'ambito dell'accoglienza dei richiedenti asilo; Paolo Boccagni, sociologo e consigliere direttivo di ATAS, ha partecipato alla valutazione del progetto INTEREST. 
certo ambiente o tra certe persone) o penalizzato dalla loro assenza; che si rivela non tanto un luogo naturale e immutabile, quanto l'esito di forme e routine quotidiane di appropriazione dello spazio, o del sentirsi più o meno a proprio agio - sicuri, familiari, in controllo - nei luoghi, domestici e pubblici, in cui si vive.

Da questa prospettiva di "fare-casa" (homemaking) interattiva, processuale e multiscalare (Boccagni, 2017a; 2017b) derivano letture originali dei processi di insediamento locale degli stranieri. In questi processi si combinano la ricerca di nuove forme di casa e di sentirsi-a-casa tra i neo-arrivati, anche al di là degli spazi domestici in cui essi vivono, e percezioni di estraneità verso spazi pubblici come quelli del quartiere in cui si risiede, abitualmente vissuti come casa-propria, in una parte degli autoctoni. Questa tensione, apparentemente astratta, sfocia in concretissime percezioni di insicurezza negli spazi pubblici, fino alla desertificazione degli stessi. Tali processi si accompagnano a problemi oggettivi di degrado urbano e marginalità abitativa e ne ingigantiscono le conseguenze.

La possibilità di ricostruire un senso di casa più aperto e inclusivo, dentro contesti locali eterogenei, merita maggiore attenzione nel dibattito e nelle politiche pubbliche, anche per la sua valenza applicativa. Nel nostro contributo rileggiamo criticamente, in quest'ottica, gli esiti di alcune iniziative di lavoro di comunità di un'associazione specializzata di Trento (ATAS onlus), che ha messo le questioni del "sentirsi a casa" al centro della propria riflessione operativa (ATAS, 2014) ${ }^{1}$. A partire dal dibattito sulla dimensione territoriale/locale dei flussi migratori, e sulla "estendibilità territoriale" del concetto di casa, rivisitiamo alcune azioni di sviluppo di comunità in aree urbane a elevata incidenza di lavoratori stranieri e, più recentemente, di richiedenti protezione internazionale. Tali iniziative erano orientate a facilitare l'interazione tra neo-arrivati e lungo-residenti, attraverso attività coprogettate di interesse locale. Hanno quindi richiesto significativi processi di riflessione e sperimentazione sui modi di percepire, utilizzare e condividere gli spazi comuni di quartiere.

\footnotetext{
${ }^{1}$ L'Associazione Trentina Accoglienza Stranieri (ATAS onlus) è stata fondata nel 1989 per affrontare il nascente fenomeno dell'immigrazione attraverso la gestione di alloggi, sportelli informativi, servizi di consulenza e iniziative per la promozione della convivenza pacifica e della cultura dell'accoglienza. A partire dagli anni 2000 ha iniziato a gestire il servizio alloggiativo in termini di accompagnamento sociale all'abitare. Dal 2013 ha portato l'approccio del lavoro di comunità in alcuni dei propri servizi abitativi e in altri progetti specifici, quale elemento fondamentale per l'integrazione e per la coesione sociale della comunità.
} 
Alla luce delle indicazioni sostanziali e metodologiche raccolte sul campo, mostriamo come la costruzione di spazi di interazione non evitante o forzata tra autoctoni e stranieri richieda di prendere sul serio le visioni e i vissuti emotivi di casa degli uni e degli altri: che cosa significhi casa e quali aspetti dei loro ambienti di vita siano percepiti come favorevoli a tali significati o incompatibili con essi, al di là delle loro cornici discorsive e valo-

riali. È dal tentativo di rispondere a diverse sfumature quotidiane del farecasa, oltre che dalla disponibilità di infrastrutture adeguate e alleanze territoriali efficaci, che dipende la capacità inclusiva e la sostenibilità degli interventi di sviluppo di comunità in contesti territoriali multietnici.

\section{Immigrazione straniera, territorialità e fare-casa}

Esiste crescente consapevolezza, nel dibattito sull'integrazione dei cittadini stranieri, circa l'importanza della dimensione territoriale dei loro processi di insediamento, e di uno sguardo analitico sensibile al livello locale.

Diversi fattori contribuiscono alla particolare rilevanza della dimensione locale, tanto per lo studio dell'immigrazione quanto per l'intervento politico e sociale. Il primo è eminentemente descrittivo: la diffusività territoriale dei processi di insediamento abitativo e lavorativo degli stranieri, irriducibili sia alle aree di confine di primo arrivo, sia agli snodi centrali delle grandi aree urbane (vedi ad esempio, per l'Italia, Osti, Ventura, 2012; Semprebon et al., 2017). Accanto a questo, la dimensione locale è cruciale per affrontare gli squilibri che tendono a prodursi nella gestione politica dell'immigrazione straniera. Se i suoi "benefici" sono più facilmente apprezzabili su scala aggregata (il contributo economico-produttivo e fiscale della popolazione straniera, o il saldo demografico positivo che essa produce), i relativi "costi" sociali in termini di vulnerabilità, bisogni insoddisfatti e possibili tensioni interetniche sono assai più visibili - benché a volte ingigantiti strumentalmente - sulla scala micro delle comunità locali. Molta letteratura recente, anche in Italia (Ambrosini, 2010), ha inoltre enfatizzato il grado di autonomia di cui godono le amministrazioni locali nell'attuare politiche per gli immigrati di taglio più o meno escludente o inclusivo. Se l'importanza del livello locale di policy-making è stata riconosciuta in tutta Europa (Zapata et al., 2017), in un contesto di ricezione (e in un regime occupazionale e di welfare sociale) ad alta variabilità locale, come quello italiano, si fa ancora più importante guardare alle specificità dei contesti locali di insediamento e alla struttura di opportunità che essi racchiudono (Barberis, Boccagni, 2017). 
Che la dimensione locale faccia la differenza per i processi di integrazione degli immigrati è un dato riconosciuto in molta letteratura recente, di taglio soprattutto etnografico, in Italia (ad es. Cancellieri, Ostanel, 2015; Marzorati, Semprebon, 2015) e altrove (ad es. Wessendorf, 2010; Sigona et al., 2014; Hall, 2012). Il ruolo delle infrastrutture, delle istituzioni e delle tradizioni di accoglienza a livello di città e perfino di quartiere, le diverse visioni della diversità (etnica e non solo) che si confrontano in un territorio, e l'andamento delle interazioni quotidiane tra maggioranze e minoranze sono tre aree tematiche privilegiate per questa prospettiva di studi. Al tempo stesso, lo studio dei rapporti tra neo-arrivati e lungo-residenti su scala micro ha importanti ricadute applicative per le politiche di welfare sociale orientate alla "comunità". Quest'ultima viene sovente evocata come ambito, interlocutore e oggetto privilegiato di interventi tesi a promuovere dal basso reciprocità, partecipazione civica, coesione sociale (Devastato, 2016). Per quanto riguarda gli stranieri, in particolare, il riferimento alla comunità - pur non esente da aspetti problematici - può rispecchiare un investimento su forme di integrazione locale che vadano al di là dell'emergenza, del breve periodo, o del soddisfacimento dei bisogni primari.

A metà strada tra il livello della ricerca accademica e quello dell'intervento sociale, l'ottica del fare-casa ambisce a essere di più che una metafora evocativa. L'idea ad essa sottesa è che sondare le idee e le pratiche di casa - se, dove, come e con chi un individuo o una famiglia si sentano "a casa" - sia una modalità efficace e non intrusiva di cogliere le conseguenze della migrazione sull'esperienza di vita quotidiana: sia nei vissuti degli stranieri, sia tra gli autoctoni che si confrontano quotidianamente con loro.

Al tempo stesso, i contorni concettuali ed emotivi dell'idea di casa, nell'esperienza della maggior parte delle persone, non si esauriscono nello spazio abitativo. È invece possibile che un senso di casa, o di (non) sentirsi a casa, si associ anche a scale territoriali più ampie, compresa quella del quartiere o della comunità locale di riferimento. Quanto più il luogo in cui si vive assume contorni diversi da quelli di prima - o, agli occhi dei neoresidenti, appare uno spazio nuovo ed estraneo - tanto più è importante cogliere gli elementi e le condizioni che lo potrebbero rendere più vicino a un'idea di casa, ovvero a uno spazio che offra sicurezza, familiarità e un certo senso di "controllo" su ciò che ci sta intorno (Duyvendak, 2011; Boccagni, 2017a; Miranda, 2018).

In pratica, tuttavia, qualsiasi contesto abitativo locale può racchiudere visioni diverse, e anche contrapposte, di ciò che fa sentire a casa, e di come sentirsi a casa negli spazi pubblici. La "desertificazione" di tali spazi è ciò che avviene nel momento in cui essi generano senso di estraneità, insicu- 
rezza, o per lo meno indifferenza, e alimentano forme di domesticità più esclusive e privatizzate.

Chiedersi dove ci sente a casa, rispetto a qualsiasi spazio pubblico o privato, vuol dire investigare come si gestisce la tensione dialettica tra gli spazi e i confini di ciò che è privato, pubblico e comune, con le rispettive soglie di passaggio e di accessibilità (Boccagni, Brighenti, 2017): da quelle più visibili e tangibili (muri, cancelli, divieti di accesso, orari di accesso) a quelle immateriali, non immediatamente percepibili ma spesso altrettanto cogenti (come la percezione diffusa di certi spazi come più adatti e appropriati per alcune pratiche che per altre, o per alcuni profili di utenti più che di altri, anche a seconda dei momenti della giornata).

Esiste una dimensione ascrittiva della estendibilità dell'idea e del senso di casa legata allo spazio pubblico - quella per cui, da tanto più tempo si risiede in un luogo (o comunque da più tempo di "altri"), ci si sente "naturalmente" più inclini a percepire quel luogo come casa (Dovey, 1985). Esiste però, accanto a questo radicato modo di sentire, una dimensione costruttiva, acquisitiva e orientata al futuro di questo stesso processo: casa non come spazio di vita che si possiede o si controlla già, ma che si può costruire in forme più o meno aperte e inclusive, nella misura in cui esso genera interesse, attaccamento, investimento nel futuro (Jacobson, 2009). Questa seconda accezione, che rimanda precisamente al fare casa, è la più promettente per le pratiche di intervento sociale; senza dimenticare che la visione ascrittiva, escludente, orientata a ripristinare un passato reale o mitizzato rimane pervasiva ed esercita conseguenze reali.

\section{Dal lavoro sociale di comunità al fare-casa}

Con queste premesse, l'ottica del fare-casa può essere applicata in modo originale al lavoro di comunità attraverso alcune pratiche di intervento di ATAS onlus nei quartieri multietnici di Trento. L'Associazione ha cercato di declinare il "sentirsi a casa" con gli strumenti dello sviluppo di comunità, teso alla promozione del benessere collettivo e a far crescere comunità competenti e contesti più abitabili per tutti. Tale approccio si basa su strategie di intervento collaborative, in cui le persone coinvolte non sono considerate utenti o clienti di un servizio, ma attori consapevoli e in grado di contribuire a farsi carico dei propri problemi (Branca, Colombo, 2003a). Al centro del lavoro di comunità c'è la promozione di un senso di appartenenza al contesto locale, di cittadinanza attiva, e quindi lo sviluppo di forme di corresponsabilità e di "proprietà" rispetto ai bisogni e ai problemi locali, 
guidate dalla possibilità di incidere sul contesto per produrre dei cambiamenti (Branca, Colombo, 2003a; Martini, Torti, 2013).

Così intesa, l'ottica del lavoro di comunità non si riduce a uno sguardo nostalgico verso il passato, alla ricerca di forme comunitarie che non esistono più (Raineri, 2012), ma mira a «(ri)trovarne il senso e (ri)costruire quei legami sociali capaci di contrastare la frammentazione sociale e l'isolamento individuale senza concedere nulla alla sua idealizzazione» (Martini, Torti, 2013: 13). Non trascura neppure il fatto che la comunità non è solo un luogo sicuro o una fonte di risorse e opportunità, ma può essere anche la culla di forme di «localismo di tipo difensivo, che concepisce il locale come un'istanza di protezione delle specificità del proprio territorio (culturali, etniche, linguistiche, economiche) dalle minacce portate dall'esterno» (Ripamonti, 2006: 1).

In questa cornice, ATAS ha promosso un insieme di pratiche tese all'equilibrio tra salvaguardia delle comunità preesistenti (in termini di identità, abitudini, diritti e doveri reciproci) e apertura alla diversità introdotta dai neo-arrivati stranieri nelle relazioni della vita quotidiana. All'interno della prospettiva dello sviluppo di comunità, nel lavoro dell'Associazione assumono un ruolo cruciale i processi di empowerment, che consentano «la creazione di legami tra le persone, i gruppi e le organizzazioni che condividono uno stesso problema e un più marcato incremento del capitale sociale di una determinata comunità locale» (Ripamonti 2006: 3). Si tratta di un approccio mirato alla promozione di spazi di incontro e confronto e di relazioni fiduciarie, al fine di ridurre la diffidenza, la conflittualità e le difficoltà di relazione tra famiglie di nazionalità diversa; ma anche, in positivo, per promuovere iniziative partecipate, aumentare il senso civico e il reciproco rispetto.

Nell'ottica di questo articolo, quindi, la comunità non è definita da una dimensione territoriale o da una gamma di interessi particolari, bensì dall'orientamento a fare interagire tutti gli attori potenzialmente rilevanti per gli obiettivi di un progetto locale: i cittadini "attivi", i vicini di casa, gli abitanti del quartiere, i negozianti, le associazioni, le istituzioni locali ecc. Tra questi soggetti si cerca di coinvolgere anche le persone più diffidenti che si trovano nondimeno a confrontarsi con nuovi vicini di casa stranieri. 


\section{Fare comunità e fare-casa in contesti multietnici e di nuova immi- grazione: due studi di caso}

Analizziamo ora due casi di lavoro di comunità realizzati da ATAS, alla luce dell'ottica del fare-casa discussa in precedenza. Nel primo di essi, l'approccio dello sviluppo di comunità è stato utilizzato per favorire l'integrazione tra i cittadini di alcuni quartieri di Trento. L'integrazione non è stata assunta come tentativo di ridurre le distanze tra culture, ma come ricostruzione di un tessuto di cittadinanza e di appartenenza aperto e inclusivo. Il senso del lavoro è stato coinvolgere alla pari cittadini italiani e stranieri cercando di far emergere bisogni e interessi concreti dalla convivenza quotidiana, dentro precisi contesti abitativi. L'obiettivo è stato dunque creare nuove relazioni di comunità e rafforzare quelle già esistenti. Nel secondo caso che analizziamo si è operato partendo dalla centralità delle relazioni per avviare percorsi di inserimento positivo dei richiedenti asilo e avvicinare la società ospitante alla realtà dell'accoglienza.

I due casi sono esemplificativi di un orientamento processuale alla integrazione, come possibilità concreta di sentirsi a casa nei luoghi in cui si vive, grazie a relazioni di prossimità più aperte e inclusive. Sotto l'ampia e generica etichetta di coesione sociale, i progetti hanno perseguito obiettivi pragmatici ma non per questo irrilevanti, come la creazione di spazi e opportunità in cui vicini di casa non abbiano paura o fastidio di incontrarsi, o si salutino e riconoscano reciprocamente - specie tra italiani e stranieri - nei luoghi della vita quotidiana.

Studio di caso - 1: Sviluppo di comunità in due quartieri periferici a elevata incidenza multietnica

La prima esperienza riguarda l'avvio di processi di sviluppo di comunità in due quartieri semiperiferici di Trento. Si è trattato di un processo di ricerca-azione ${ }^{2}$, attivazione di relazioni, formazione, coprogettazione e lavoro di gruppo. Tali attività sono state rese possibili da due finanziamenti europei del Fondo Europeo per l'Integrazione (FEI), i progetti TRA.Mi.Te e

\footnotetext{
${ }^{2}$ La ricerca-azione nello sviluppo di comunità è la metodologia costitutiva dei processi di empowerment. È una ricerca che si immerge nella comunità, favorisce la presa di coscienza dei bisogni e problemi da parte della comunità, accompagna a farsene carico a partire da nuove ipotesi di sviluppo attraverso la progettazione partecipata (Branca, Colombo, 2003b).
} 
INTEREST (settembre 2013 - luglio 2015) ${ }^{3}$. Entrambi i progetti erano orientati alle comunità di quartiere nel loro complesso più che ai soli cittadini stranieri. Attraverso iniziative coprogettate e attuate con i cittadini si è lavorato per rafforzare le relazioni e le reti tra individui e associazioni, con un buon coinvolgimento sia di lungo-residenti stranieri, sia di richiedenti asilo di recente arrivo.

Il primo ambito urbano considerato è il quartiere di Solteri-Centochiavi nell'area nord di Trento. Si tratta di un quartiere semiperiferico in cui la percezione di disagio sociale è legata all'assenza di spazi comuni di aggregazione, formali o informali: sale, infrastrutture pubbliche, o anche solo piazze. Questo limita notevolmente le occasioni di incontro e socializzazione. I servizi presenti sul territorio (uffici, centri commerciali, ecc.) sono fruiti soprattutto da persone esterne al contesto e quasi solo durante la settimana. Gli abitanti del quartiere, dice un testimone, «non si vedono». L'eterogeneità del tessuto urbano e del tipo di alloggi a disposizione comporta un contesto diversificato e frammentato in termini di appartenenza sociale. Difficile attendersi che nascano spontaneamente relazioni di comunità in un'area che, sottolinea un educatore da noi intervistato, «è nata come quartiere di servizi e non ha mai avuto uno sviluppo legato alle persone». Il secondo quartiere considerato, le Torri di Madonna bianca, è un complesso di palazzi di edilizia abitativa risalente agli anni Sessanta. La quotidianità alle Torri è fatta soprattutto di persone, come pensionati, disoccupati, casalinghe e bambini, che trascorrono nel quartiere la maggior parte del tempo, ma senza interagire più di tanto con e nel territorio. Più che di un quartiere "dormitorio" potremmo parlare di un quartiere "assopito".

La maggior parte delle persone intervistate dichiara di "vivere bene" in entrambi i contesti. Questa espressione, a rileggere bene le narrazioni, racchiude in sé aspetti diversi e contrastanti:

- c'è chi proviene da altrove e ha provato a instaurare qualche legame per avere un senso di familiarità, senza successo;

- non conoscere nessuno o non avere rapporti di vicinato comporta un senso di insicurezza nel contesto in cui si vive, ancor più se si è stranieri;

\footnotetext{
${ }^{3}$ Con il progetto TRA.Mi.Te si è lavorato principalmente sulla dimensione di prossimità a partire da alcuni condomini, riuscendo a far nascere nuove relazioni e reti informali, in grado di far sentire le persone "più a casa" e favorire il senso di appartenenza in un dato contesto abitativo. Con il progetto INTEREST c'è stata una transizione dal lavoro di vicinato al lavoro di quartiere, che ha permesso un maggiore coinvolgimento delle realtà associative e dei gruppi di cittadini attivi. Durante la prima fase della ricerca-azione sono state fatte 46 interviste a testimoni privilegiati e 54 a membri di famiglie residenti (21 stranieri e 33 italiani, di cui 4 sinti e rom).
} 
- si avverte un senso di tensione nei condomini e di frustrazione tra chi si è sempre preso cura del contesto, a fronte del minore interesse verso il bene comune percepito nei nuovi arrivati;

- la poca comunicazione tra le persone, legata alla scarsa conoscenza reciproca, ha una certa influenza sulla conoscenza delle regole e la loro condivisione. I nuovi arrivati trovano spesso il vuoto, non sviluppano un senso di appartenenza verso il contesto e lo vivono con maggiore distacco;

- prevale la tendenza a lamentarsi e ad attribuire tutto ciò che non funziona all'Ente per l'edilizia abitativa. Al di là della dimensione del condominio, si registra scarsa adesione alle iniziative organizzate dalle associazioni locali.

Si tratta quindi di un "vivere bene" centrato sulla dimensione protettiva, intima e privata della casa, ma influenzato anche da un contesto fisico e relazionale sfavorevole.

In questo quadro, l'insediamento crescente di persone straniere è oggetto di percezioni difformi. A Solteri-Centochiavi (caso 1) tale presenza, relativamente alta, non emerge come elemento caratterizzante tra gli abitanti intervistati, come se si perdesse nell'indifferenza più generale. Alle Torri (caso 2) si avverte un forte scollamento tra un prima, sino agli anni Novanta, in cui tutti i residenti erano autoctoni e c'era una forte solidarietà legata a bisogni comuni e a forme di autogestione del condominio e del quartiere; e un dopo, che arriva fino a oggi, in cui le relazioni sono meno buone, ognuno preferisce restare dentro il proprio appartamento e le nuove famiglie giovani con bambini, quasi tutte straniere, vengono considerate maleducate, rumorose, poco interessate al bene comune. Nelle narrazioni degli intervistati il cambiamento rispetto al passato coincide con l'arrivo e la crescente presenza di stranieri. Così, ad esempio, nella testimonianza di un inquilino italiano:

il fatto è questo, negli anni '80 quando hanno consegnato gli appartamenti eravamo tutti trentini, italiani e tutti giovani, 30 anni, 35, perciò si andava d'accordo tantissimo. Però piano piano, si è sempre andati d'accordo, però alcuni hanno cambiato casa, i figli cresciuti sono andati da un'altra parte, sono arrivati gli extracomunitari, perciò c'è stato un cambiamento. Grosso anche. Non ci sarà più quel modo... nei primi anni ci si trovava a bere da basso. Ognuno portava del vino...si faceva el filò. Adesso quelle cose lì non si fanno. Ognuno sta dentro dalla propria porta (testimonianza di un inquilino italiano di vecchia data).

In quasi tutte le interviste con gli inquilini italiani emerge un certo disagio rispetto all'arrivo dei cittadini stranieri. Sembra che il problema sia 
rappresentato dalla loro stessa presenza, più che da loro comportamenti. Le occasioni di reciproco incontro, confronto ed eventuale conflitto sono scarse. Prevale l'immaginario consolidato di una presenza estranea, sconosciuta e pertanto da temere, che sembra ostacolare l'interazione interetnica. Ovviamente questo atteggiamento di chiusura e diffidenza viene percepito dagli stranieri:

la gente nelle assemblee parla sempre degli stranieri male, che sporcano, urinano, che non sono educati, anche se i miei figli non sono mai andati giù da basso... che buttiamo le sigarette anche se noi non fumiamo, i musulmani di qua non fumano. Gli italiani non ci salutano e allora meglio che non li saluto, la prima volta saluto, la seconda saluto e poi basta non saluto più. Quando succede qualcosa dicono sempre che sono stati gli stranieri e io invece non faccio niente. Ho paura a muovermi perché penso sempre che pensano che sono io o i miei figli che facciamo le cose (testimonianza di un'inquilina marocchina).

A questo distacco tra due mondi contribuisce il divario generazionale e di condizione abitativa. Quasi tutti i residenti italiani sono anziani, spesso soli, mentre gli stranieri sono giovani e con famiglie numerose. La maggior parte dei cittadini italiani è proprietaria degli alloggi o affittuari di lungo periodo, mentre i cittadini stranieri spesso vivono la propria presenza come temporanea. Ciò che accomuna entrambi è la nostalgia per un passato - per gli uni alle Torri, per gli altri nel paese di origine - in cui, si dice, le relazioni di vicinato e il vissuto di comunità erano più forti. Da ambedue i versanti, l'attribuzione di un certo senso di casa allo spazio di quartiere è tutta orientata al passato, mentre rimane debole, o inesistente, al presente.

A partire dal quadro relazionale emerso durante la prima fase della ricerca-azione, il progetto di ATAS ha dapprima rivolto attenzione alle relazioni di condominio e di prossimità, per poi investire sulle reti di quartiere, favorendo il coinvolgimento di cittadini italiani e stranieri. Le principali azioni in questo senso sono state i micro incontri e momenti conviviali a livello di condominio; poi i caffè dibattito, quale occasione di convivialità e confronto; infine brevi percorsi formativi, iniziative di quartiere e laboratori co-progettati.

La ricognizione sociale ha favorito la nascita di relazioni tra persone di uno stesso condominio che, pur di estrazione assai diversa (un'anziana signora italiana, un signore cileno con famiglia e un gruppetto di sette rifugiati dell'Africa Subsahariana), vivevano condizioni molto simili di solitudine e di fatica a relazionarsi con il contesto: 
a me piacerebbe, ho provato con due o tre che sono qui, ho provato un primo approccio con delle vicine per fare qualcosa insieme, però mi hanno detto che loro stanno bene così. Non trovi agganci. Ho provato a cercare il contatto con delle persone. Però qua in casa, le persone anziane, proprio non cercano il contatto, io per esempio che sono nuova, ho cercato di farmi dentro. Quando entri in una famiglia nuova, perché per me una casa è una famiglia, ho cercato di essere carina a dir ciao, come va, a dire buon giorno. Ma intanto lei che abita qua come si trova? Anche per avere delle informazioni perché io ero nuova. Però qua ti guardano male se fai una domanda in più (testimonianza di una signora anziana italiana residente da sola da 5 anni).

È una bellissima zona e dirò che è una bellissima città, la città è bella. Però una città non può essere bella finché non ci sono relazioni tra i cittadini e persone straniere. Perché non so perché però quello che ho capito la gente hanno un po' di paura, magari può essere paura, magari hanno delle difficoltà a mettersi in contatto tra la gente... In questo condominio sono da quasi tre mesi, ma non posso dire di avere fatto amicizia con qualcuno... Ti salutano appena e se sali insieme sull'ascensore non ti parla nessuno... Tipo se tu mi hai parlato io sono contento, perché tu mi hai dato un valore che io sono umano, e che tu non ti sei messo in una posizione superiore rispetto a me, di superiorità, quindi sono fiero di questa cosa e sono contento di chiacchierare con te... (rifugiato del Mali di 30 anni in Italia da 2 anni e mezzo).

A partire da quanto emerso dalle interviste, strumento non solo di esplorazione ma anche di riattivazione, è stato possibile favorire l'incontro tra un gruppetto di persone che ha poi iniziato a frequentarsi regolarmente una volta alla settimana. Un primo incontro ha avuto luogo tra un condomino italo/cileno e alcuni rifugiati africani. Durante l'intervista il primo è venuto a sapere della presenza dei rifugiati: non ricordava di averli mai incontrati o di aver fatto caso alla loro presenza. Quello che doveva essere un breve momento di incontro si è trasformato in una lunga conversazione in cui il signore cileno ha raccontato ai rifugiati il percorso migratorio degli antenati in Cile all'inizio del '900, e il rientro in Italia negli anni 2000. Per una volta i rifugiati, che solitamente ricoprono la parte di testimoni del proprio viaggio, hanno ascoltato, con sorpresa, la storia di qualcun altro. A partire da quel momento i rifugiati si sono di volta in volta fermati a chiacchierare con lui e la sua famiglia, e hanno espresso la soddisfazione di avere un punto di riferimento in più nel proprio contesto abitativo.

Il secondo incontro è nato dal desiderio, espresso dalla vicina dei rifugiati durante l'intervista, di conoscere meglio i nuovi vicini di casa, dei quali si era fatta un'immagine molto positiva senza riuscire ad andare oltre il saluto. L'incontro, facilitato dall'operatrice di comunità, ha visto la partecipazione di tutti i rifugiati, della vicina italiana e di un'altra coppia dello 
stesso condominio. Nelle settimane successive è diventato un appuntamento fisso promosso spontaneamente dalle persone coinvolte. In seguito la signora italiana avrebbe ricordato quel periodo come uno dei più belli della sua vita. Per i rifugiati è stata l'occasione per sentirsi, per alcuni di loro per la prima volta dall'arrivo in Italia, un po' più "a casa" in un contesto abitativo per certi versi ostile.

Tra le azioni messe in campo nei due anni di progetto, il caffè-dibattito è stato per molti cittadini stranieri l'occasione per conoscere nuovi vicini e uscire dalla routine quotidiana, ma anche essere ascoltati. I temi emersi hanno spesso riguardato la relazione tra stranieri e italiani all'interno di uno stesso contesto - il saluto, le diversità culturali nella gestione della casa, gli odori del cibo, il diverso valore dato al bene comune - e ha permesso attraverso il confronto, anche teso, di ridurre le distanze percepite. Accanto alle differenze è sempre emerso il bisogno di relazioni e di comunità che le persone nei due contesti sentivano di avere. In alcuni casi, il processo di conoscenza reciproco avviato con i caffè dibattito ha avuto esiti di lungo periodo, in termini di nuove reti di supporto reciproco.

$\mathrm{Al}$ tempo stesso, gli incontri di progettazione hanno portato intorno allo stesso tavolo un gruppo molto eterogeneo, composto da donne e uomini anziani, presenti da lunga data nel territorio; da adolescenti italiani e non; da richiedenti asilo e giovani stranieri con figli piccoli, di diverse nazionalità; da persone con la stessa nazionalità, ma con un modo diverso di interpretare la propria cultura d'origine. Se questo ha reso possibile una varietà di contributi, non sempre ha facilitato il dialogo. In alcune occasioni il rapporto tra italiani e stranieri, pur non essendo l'oggetto degli incontri, è emerso come fonte di sottili barriere. Proprio lo "stare insieme" nella diversità, però, è servito a evitare che le barriere diventassero muri, e ha reso possibile vivere esperienze positive che rimuovessero almeno in parte le reciproche percezioni negative. Nei momenti più delicati è stato proprio il contributo dei singoli a far sì che pregiudizi e diffidenza venissero messi da parte.

Sul piano degli esiti, a livello di comunità, le iniziative di progetto hanno reso possibile il consolidamento di un piccolo gruppo di persone, italiane e straniere, con un certo interesse per il territorio e le relazioni, e con obiettivi concreti in comune. I momenti di incontro hanno avuto effetti tangibili, come la partecipazione locale, ma anche meno tangibili, sul piano dei vissuti personali, avviando processi di cambiamento di pensiero e approccio rispetto alla presenza degli stranieri sul territorio.

Sul piano dei rapporti di prossimità, era stato più volte espresso nelle interviste iniziali un senso di estraniazione rispetto al contesto, soprattutto per gli stranieri, per la quasi totale assenza di relazioni interpersonali. A fronte 
di questo, i progetti hanno generato tra le persone coinvolte un maggior senso di sicurezza e di appartenenza, come di "sentirsi di più a casa". Ciò ha mostrato quanto sia stato importante favorire i momenti di incontro a livello di condominio, stando in prima persona dentro le relazioni e accompagnando un processo di conoscenza reciproca che può apparire semplice e scontato, ma di fatto necessita di un "tramite" qualificato. È grazie a questi tramiti che si può realizzare, con il tempo, il passaggio da una visione di casa protettiva - circoscritta all'abitazione, in opposizione a ciò che le è esterno - a una visione più aperta e inclusiva, basata su interazioni positive, e di reciproco rispetto, tra vicini.

Al tempo stesso non vanno trascurate le criticità riscontrate nei due progetti, anzitutto per il coinvolgimento dei cittadini stranieri in momenti di formazione e di riflessione, funzionali anche alla coprogettazione. A livello delle relazioni di prossimità la maggiore difficoltà è il coinvolgimento dei cittadini stranieri di religione musulmana in forma non differenziata: raramente si riesce a coinvolgere uomo e donna insieme, se non pensando a iniziative diverse e in orari diversi. Ci sono poi rilevanti diversità di interessi, orari, aspetti legati alla gestione familiare, nonché di ordine linguistico. Se gli uomini (o i mariti) erano tipicamente più difficili da "intercettare", il contatto con le donne era relativamente agevole nei "loro" spazi abituali (parchi, doposcuola, ecc.), ma più difficile in iniziative organizzate al di fuori di tali spazi. Particolarmente importante, per il miglioramento delle relazioni di vicinato, è stata la partecipazione a momenti comunitari e trasversali alle appartenenze etniche o nazionali.

\section{Studio di caso - 2: Lavoro di comunità e accoglienza dei richiedenti asilo}

Con l'apertura di un centro di accoglienza per 70 giovani richiedenti asilo di sesso maschile nel quartiere dei Solteri, a partire da settembre 2014, l'approccio del lavoro di comunità si è esteso all'accoglienza straordinaria promossa dalla Provincia autonoma di Trento. Il progetto della struttura, denominata Residenza Brennero, era finalizzato non soltanto ai normali obiettivi di integrazione civica (corsi di lingua, pre-orientamento lavorativo ecc.), ma anche a far vivere gli spazi di quartiere come punto di riferimento per il periodo di accoglienza (Di Battista, Volpato, 2016). In quest'ottica la comunità è stata considerata come risorsa per agevolare l'individuo nel raggiungimento di una piena autonomia e consapevolezza del contesto in cui vive, ma anche come luogo in cui sentirsi "più a casa", o quanto meno guadagnare spazi di autorealizzazione e crescita personale. 
Al di là della risposta ai bisogni dei richiedenti come utenti di un servizio, c'è stato quindi il tentativo di coinvolgerli come abitanti del contesto locale. Questo è stato possibile solo nel tempo, sia per aspetti legati al percorso dei richiedenti stessi, sia per le caratteristiche della comunità locale, alquanto disgregata e non sempre aperta al cambiamento. La promozione di legami tra i richiedenti asilo e i lungo-residenti, facilitata dal radicamento degli operatori nel quartiere, è servita anche a favorire una diversa percezione dell'accoglienza. Ciò ha a sua volta permesso ai richiedenti di sentirsi meno estranei alla vita di quartiere.

Un esempio in questi termini può essere dato dalla collaborazione con le associazioni, a partire da una festa organizzata in avvio del progetto. Questo singolo episodio non è stato sufficiente per coinvolgere i richiedenti asilo nelle iniziative delle associazioni, che, come il quartiere nel suo insieme, hanno vissuto l'apertura del centro con perplessità e distacco. Un primo cambiamento si è registrato nel momento in cui i richiedenti asilo sono stati coinvolti direttamente negli incontri organizzativi di una nuova festa di quartiere. La struttura di accoglienza, considerata estranea e portatrice di problemi più che di risorse, ha iniziato a essere vista diversamente. Negli anni, attività inizialmente proposte dall'operatrice sono state poi promosse direttamente dai cittadini e, a volte, maggiormente strutturate grazie all'aiuto dei richiedenti asilo stessi ${ }^{4}$. Quello che più interessa però, non è tanto la quantità di iniziative che hanno visto il coinvolgimento dei richiedenti asilo, ma aspetti di processo che si riscontrano anche in altri territori in cui ci si ispira al lavoro di comunità:

- la possibilità di partecipare, attraverso la vita di quartiere, ad iniziative utili al percorso di autonomia personale;

- l'opportunità per i richiedenti asilo di sentirsi meno estranei al contesto e più efficaci nell'orientarsi in esso, e nell'usufruire delle risorse disponibili;

- il cambiamento nella percezione collettiva del fenomeno della accoglienza.

In questa sede interessa approfondire il secondo e il terzo punto. Durante il progetto si è cercato di osservare che impatto potesse avere il coinvol-

\footnotetext{
${ }^{4}$ Tra queste si possono ricordare il coinvolgimento degli ospiti nelle varie iniziative di quartiere; l'organizzazione di attività ad hoc con loro (per esempio gite in montagna); l'inserimento dei richiedenti asilo come volontari in attività di lungo periodo (banco alimentare, doposcuola, gruppo di cucito); l'avvio di iniziative di quartiere pensate per favorire il coinvolgimento di richiedenti asilo e promuovere lo scambio interculturale (workshop musicale sulla musica africana; concerto africano durante la festa di quartiere), la partecipazione alla più ampia vita di comunità.
} 
gimento nella vita di quartiere sulla quotidianità dei richiedenti asilo. Questi ultimi, in un'iniziativa della Giornata internazionale del Rifugiato del 2015, hanno sottolineato gli aspetti positivi della loro partecipazione alla vita di quartiere, non solo come soluzione pragmatica per "riempire il tempo" nei mesi (o anni) di attesa della decisione sul loro status.

Perché qui è bene... perché noi stiamo tutto il giorno in camera con tanti pensieri a non fare niente. Ma l'operatore viene a chiamarci per attività e possiamo incontrare persone italiane (giovane del Gambia, in Italia da un anno circa).

Noi diciamo grazie al Trentino, però non è sempre facile, perché le persone non sono sempre gentili. Quando siamo sul bus molte si alzano se ci sediamo noi... però qui abbiamo la possibilità di conoscere le persone del quartiere. C'è la "capa" (ride... è così che chiama l'operatrice che si occupa delle relazioni di comunità) che ci invita a partecipare. Stiamo insieme alle persone italiane e facciamo attività e cose utili e poi le persone ci salutano se ci vedono per strada. Questo è molto importante (giovane del Niger, in Italia da due anni circa).

Tutti gli ospiti della Residenza Brennero, inoltre, arrivavano da un altro centro, dove alcuni mesi prima erano stati accusati di avere partecipato a uno stupro. L'accusa, presto rivelatasi infondata, aveva portato al prelievo del DNA di tutti loro, atto considerato fortemente discriminatorio. Ne era derivato un forte senso di insicurezza e un certo timore a uscire dal campo. Trovarsi in una situazione opposta, con un progetto che favoriva l'inserimento in una comunità più accogliente, ha favorito un recupero di autostima e della possibilità di sentirsi parte del nuovo contesto di vita ${ }^{5}$. In generale, per persone che hanno vissuto forme di sradicamento lungo tutto il percorso migratorio, riconoscersi ed essere riconosciute nella comunità non è solo fondamentale per un inserimento positivo, ma è anche utile al percorso di autonomia, ovvero a rafforzare competenze e motivazioni spendibili in futuro e altrove. Questo viene confermato anche da ex ospiti ora in possesso di permesso di soggiorno e di un lavoro, che vivono in autonomia in un alloggio sul territorio. Avendo trovato un'occupazione sono senz'altro più fortunati di altri, ma spesso non hanno relazioni e occasioni di coinvolgimento nel tempo libero. Un ex ospite della Guinea Conakry, attualmente panettiere a Trento, si è espresso così in un incontro di sensibilizzazione nelle scuole:

\footnotetext{
${ }^{5}$ Questo aspetto è stato evidenziato da un'operatrice che seguiva i richiedenti asilo sia nel primo centro che nel secondo.
} 
[partecipare a questo incontro] è un piacere per me. Da quando sono uscito dal progetto non mi chiama più nessuno per partecipare a delle attività... dove abito non conosco nessuno e nel tempo libero non faccio quasi niente. Dormo, lavoro e mangio... non è bello così... dov'ero prima conoscevo delle persone e facevamo delle cose insieme (rifugiato della Guinea Conakry in Italia da 4 anni circa).

In quanto agli atteggiamenti percepiti nella comunità locale, diversi episodi segnalano la possibilità di attivare cambiamenti efficaci per via esperienziale - benché la loro sostenibilità nel tempo, e la loro trasferibilità a contesti diversi, siano tutte da verificare. La comunità dei Solteri non ha reagito in modo particolarmente conflittuale all'apertura della Residenza Brennero, ma sia negli ambienti delle associazioni che tra i cittadini affioravano diffidenza e malcontento. Nel corso dei mesi, oltre a coinvolgere sempre più i richiedenti asilo, è stato possibile riscontrare come i cittadini vicini all'accoglienza si siano fatti portatori di un pensiero positivo con altri più restii. Due esempi possono spiegare meglio. Il primo riguarda una piccola iniziativa organizzata dagli alpini in una giornata particolarmente fredda, durante la quale il capo degli alpini ha invitato i richiedenti ad accomodarsi al caldo all'interno del circolo. Visto che la nuova situazione ha causato malcontento tra gli avventori abituali, il capo degli alpini ha spiegato loro il motivo della presenza dei richiedenti asilo in Italia e il perché fosse giusto che lo Stato garantisse loro un progetto di accoglienza. Questa sua presa di posizione, oltre ad essere frutto di un lungo processo, è stata più efficace di qualsiasi articolo di giornale o serata pubblica per sensibilizzare la popolazione residente.

Un altro esempio riguarda una manifestazione di un gruppetto di richiedenti asilo contro il sistema di accoglienza, che ha visto l'occupazione di una delle arterie principali del quartiere per alcune ore. Le operatrici di comunità temevano che l'iniziativa influisse negativamente sul processo avviato all'interno del quartiere e portasse a una fase di stallo o di conflitto. In realtà una delle associazioni si è fatta immediatamente sentire, non per giudicare l'accaduto però, ma per dichiarare la propria solidarietà e disponibilità a testimoniare presso il Commissariato del Governo la positiva integrazione di alcuni ospiti coinvolti nella manifestazione, a rischio di espulsione dal progetto.

Ancora più interessante è mettere in luce gli effetti di reciprocità che alcune azioni di processo hanno avuto in merito ai due punti sopra evidenziati. Un esempio è l'attività di volontariato di alcuni richiedenti asilo presso il banco alimentare del quartiere. Alla proposta di coinvolgere alcuni richiedenti asilo come volontari, la principale realtà organizzatrice ha inizialmen- 
te opposto qualche resistenza legata sia alle reazioni attese dai beneficiari del servizio, sia all'incapacità di prefigurare una reale collaborazione con loro. Negli anni questo atteggiamento è mutato notevolmente. In un primo momento alcuni richiedenti asilo sono stati coinvolti solo in attività di carico e scarico della merce; in un secondo momento sono stati invitati a unirsi ai volontari nella distribuzione del cibo; in una terza fase sono stati coinvolti nell'attività nel suo complesso ed è stata ventilata l'ipotesi che si occupino in autonomia del banco alimentare in estate, nel periodo di maggiore assenza degli altri volontari. Un ulteriore cambiamento è confermato dal fatto che, mentre nella fase iniziale gli attori del territorio si relazionavano esclusivamente con l'operatrice di riferimento, nel corso dei mesi hanno iniziato a rapportarsi direttamente con i richiedenti asilo. Tra questi ultimi, chi partecipa ad attività continuative tende ad acquisire maggiore consapevolezza del contesto in cui vive, maggiore riconoscimento negli eventi pubblici di quartiere, maggiore percezione di essere accolto; più come abitante e cittadino, quindi, che solo come volontario o utente di un servizio.

Anche in questi casi, naturalmente, non sono mancate le criticità: non soltanto nel quartiere considerato ma in tutti i territori con alloggi o centri di accoglienza. Molte di queste, peraltro, hanno a che fare con la "temporalità sospesa" che è propria dei richiedenti asilo (Brekke, 2004; Rotter, 2016) più che con la loro condizione di stranieri. Basti pensare agli effetti dell'elevato turnover degli ospiti; al peso burocratico e psicologico dei documenti e dei colloqui da preparare, rispetto alle possibilità e alle motivazioni a partecipare; al rischio di privilegiare iniziative di interesse esclusivo della comunità, anziché degli ospiti; alla necessità di più tempo, da parte degli operatori sociali, per accompagnare i processi e per adattarsi al nuovo modo di lavorare; alla possibilità di coinvolgere solo poche persone in questo tipo di processo; all'impossibilità, per molti degli ospiti, di restare a vivere nei territori in cui hanno instaurato legami.

Un'ulteriore criticità rimanda a una questione sovente discussa in tema di integrazione dei richiedenti asilo, e destinata a rimanere aperta ben al di là di questi progetti. Se la partecipazione civica volontaria di una parte di loro, pur avendo ricadute oggettivamente positive, rischia di riprodurre una distinzione impropria tra utenti "meritevoli" e "non meritevoli", o di anteporre l'accettabilità sociale alla titolarità del diritto alla protezione internazionale, occorre anche consapevolezza critica delle forme di questo coinvolgimento. Sovente, i primi richiedenti asilo che si insediano in un dato quartiere si trovano a subire più di tutti gli altri atteggiamenti di distacco, diffidenza, se non discriminazione. È attraverso la relazione che si costruisce nel tempo che tali atteggiamenti possono mutare. D'altra parte il "vo- 
lontariato dei richiedenti asilo" nei progetti di accoglienza è spesso circoscritto a mansioni subordinate e stigmatizzanti, come nella pulizia delle strade, che tendono a riprodurre una percezione vittimistica o pietistica degli stessi richiedenti asilo. Che questo sia, di fatto, il modo più semplice ed efficace per creare contaminazione e incontro dal basso, non altera i presupposti "alterizzanti" delle iniziative stesse. Nella pratica del lavoro di comunità, tuttavia, i richiedenti asilo rivestono il ruolo di volontari alla pari degli altri, entro attività promosse innanzitutto dai cittadini di quel contesto, con e per altri cittadini. In questo senso anche la pulizia di quartiere, come momento di incontro e di cura del bene comune, assume un significato diverso dal coinvolgimento strumentale in lavori di pubblica utilità.

In conclusione, l'orizzonte del "sentirsi a casa" di questa progettazione si è tradotto in un duplice obiettivo, che ha tutti i contorni di un work in progress. Per gli abitanti del quartiere ricevente, l'obiettivo sta nel riconoscimento sociale positivo dei richiedenti asilo: il graduale passaggio da un quadro cognitivo emergenziale (i neo-arrivati come fonte inaudita di bisogno, ma anche di sospetto e potenzialmente di pericolo) a uno più aperto alla normalità (la presenza dei richiedenti asilo come aspetto della vita quotidiana non particolarmente problematico o rilevante; in questo senso parte di ciò che è naturalizzato come "casa"). Cruciale, al di là del ruolo delle istituzioni pubbliche, è proprio la mediazione delle reti informali di comunità. Per quanto riguarda i richiedenti asilo, la proposta di partecipazione alle iniziative di quartiere e di volontariato incontra un grado variabile di apertura, non riducibile ad aspetti strumentali (fare una buona impressione, avere più "certificati", ecc.). Almeno in alcuni casi, la partecipazione attiva dei richiedenti asilo - fatta di apprendimento linguistico, relazioni costruite, presenza nella sfera pubblica in ruoli diversi da quello di deviante o di bisognoso - segnala uno sviluppo ulteriore: il rafforzamento della motivazione e della capacità di mettersi in gioco nella relazione con la società ospitante, e quindi di "fare casa" in essa, quale che sia il contesto in cui ci si troverà a vivere dopo; e quale che sia il senso di appartenenza, o identificazione, con contesti di vita per lo più provvisori.

\section{Conclusioni: a casa, fuori casa; "case" esclusive e inclusive}

«È difficile sentirsi a casa per gli italiani», notava un'operatrice di comunità intervistata nel corso di INTEREST, «figuriamoci per gli stranieri!». L'esperienza dei progetti analizzati invita a mitigare queste affermazioni, almeno tra i gruppi (auto-selezionati e non rappresentativi) dei parte- 
cipanti attivi, e almeno per due obiettivi minimali: il superamento della diffidenza di principio verso gli stranieri, tra i lungo-residenti autoctoni; il raggiungimento di un certo grado di riconoscimento sociale (dall'abitudine al saluto con i vicini di casa al non sentirsi oggetto di sguardi stigmatizzanti), tra i neo-arrivati stranieri. Di qui la possibilità di sentirsi a casa almeno in alcuni momenti, almeno nel perimetro di reti di vicinato mobilitate ad hoc - oltre che, idealmente, nel proprio domicilio, se si ha la fortuna di averlo.

Come mostrano i progetti di ATAS, il lavoro sociale di comunità può effettivamente potenziare un senso di casa "positivo" entro contesti urbani multietnici o di nuova immigrazione. Cruciale, a questo scopo, è la diffusione di pratiche di sociabilità ed esperienze di convivenza quotidiana reiterate nel tempo, che facilitano la conoscenza diretta tra le persone e le iscrivono reciprocamente nel perimetro di ciò che è percepito come "normale"; esattamente come "normali", dal punto di vista degli autoctoni, sono le case e i territori in cui abitano. La condizione per cui ci si sente a casa nella comunità in cui si vive, anziché entro il solo spazio domestico, è mediata da percezioni situate, e facilitata da reti interpersonali inclusive, in loco. Laddove essa viene meno, o va creata daccapo, rappresenta un obiettivo che ha a che fare con la pratica tenace e paziente del lavoro sociale di prossimità, più che con dichiarazioni programmatiche o con la semplice implementazione di interventi pubblici calati dall'alto.

Nel caso considerato, e nella quota auto-selezionata dei partecipanti attivi, lo sviluppo di spazi e opportunità di interazione collaborativa tra lungo-residenti e neo-arrivati è sfociato in un "sentirsi a casa" inclusivo, basato sulla conoscenza diretta o almeno la frequentazione di spazi comuni, anziché esclusivo, relegato nella sfera domiciliare, impermeabile o ostile a differenze linguistiche, somatiche o di stili di vita. È però uno sviluppo di valenza fortemente territorializzata, legato a infrastrutture disponibili e a reti di relazioni sedimentate entro contesti specifici, anche in relazione alle politiche urbane, abitative e per gli immigrati. Alla luce di questa dimensione contestuale, non è immediata la sua trasferibilità in territori o contesti diversi.

Accanto a questo, l'indicazione centrale dei progetti analizzati è che il sentirsi a casa degli stranieri neo-arrivati, negli spazi di quartiere, è interdipendente con il sentirsi a casa di tutta la comunità locale. Non è possibile né desiderabile lavorare sui processi di integrazione locale degli stranieri senza considerare il delicato interfaccia con i processi di integrazione dei già residenti (Boccagni, Pollini, 2012). Se la qualità delle relazioni di quartiere è un aspetto discriminante del radicamento in un territorio, è difficile 
che gli abitanti si sentano "radicati" dentro rapporti di prossimità ostili o di totale separazione reciproca. Fino a che gli autoctoni o lungo-residenti percepiscono come una minaccia i nuovi arrivati (o alcuni profili di essi) nei "loro territori", e finché questo senso di proprietà (la casa in versione escludente) non viene decostruito e reso più flessibile, difficilmente esisteranno le condizioni per relazioni intergruppo più aperte e per una maggiore coesione sociale. Nel solco del lavoro sociale di comunità, occorrono interventi pragmatici e trasversali alle appartenenze ideologiche, che anziché sfociare in rituali lamentele sull'ostilità dei "nativi" si sforzino di costruire dal basso le condizioni per il suo superamento.

In conclusione, la domanda la casa di chi?, benché astratta, non è retorica né accademica. Riletta dall'interno delle relazioni di quartiere, essa fa da cartina di tornasole per reazioni contrapposte e parimenti plausibili, a seconda dei codici ideali, morali e politici che si condensano nell'idea di casa. Può generare una risposta orientata al passato e alla salvaguardia dello status quo, laddove il quadro identitario ed emotivo della casa viene esteso alla scala territoriale del quartiere, della città, o di un paese intero (Walters, 2004) come riproduzioni di un'identità autoctona semplificata e ideologicamente orientata. La stessa domanda, tuttavia, può condurre a risposte di segno inclusivo, se l'immaginario della casa rimanda a un prodotto collettivo in divenire, migliorabile, potenzialmente estendibile. Reindirizzata al futuro - al processo del fare-casa, più che alla casa come prodotto o essenza - l'immagine aiuta a pensare forme di interazione interetnica che riconoscano i diritti e i bisogni delle diverse parti in causa, in un quadro unitario. Nell'uno o nell'altro scenario, non c'è motivo di trascurare la cogenza emotiva e identitaria degli immaginari della casa e della comunità; salvo "regalarli" a istanze sociali e politiche conservatrici, fondamentalmente contrarie a relazioni interetniche più eque e inclusive.

\section{Riferimenti bibliografici}

Ambrosini M. (a cura di). (2010). Governare città plurali. Milano: FrancoAngeli.

ATAS (2014). Sentirsi a casa anche fuori casa. Report del progetto FEI TRAMITE.

Barberis E., Boccagni P. (2017). Il lavoro sociale con le persone immigrate. Rimini: Maggioli.

Boccagni P. (2017a). Migration and the search for home. Mapping domestic space in migrants' everyday lives. New York: Palgrave.

Boccagni P. (2017b). Fare casa in migrazione. Una chiave di lettura dei processi di integrazione e di riproduzione sociale quotidiana in contesti multietnici. Tracce Urbane, 1(1): 60-68. doi: 10.13133/2532-6562_1.7. 
Boccagni, Volpato

Boccagni P., Brighenti A. (2017). Immigrants and home in the making: Thresholds of domesticity, commonality and publicness. Journal of Housing and the Built Environment, 32(1): 1-11. doi: 10.1007/s10901-015-9487-9.

Boccagni P., Pollini G. (2012). L'integrazione nello studio delle migrazioni. Milano: FrancoAngeli.

Branca P., Colombo F. (2003a). La ricerca-azione come promozione delle comunità locali (inserto). Animazione sociale, 169: 29-61.

Branca P., Colombo F. (2003b). La ricerca-azione: strumenti per la fase di attivazione (inserto). Animazione sociale, 172: 27-64.

Brekke J.P. (2004). While we are waiting. Uncertainty and empowerment among asylum seekers in Sweden. ISF Report.

Cancellieri A., Ostanel E. (2015). The struggle for public space. City, 19(4): 499-509. doi: $10.1080 / 13604813.2015 .1051740$.

Devastato G. (2016). Lavoro sociale e azioni di comunità. Rimini: Maggioli.

Di Battista F., Volpato S. (2016). Il lavoro di comunità nell'accoglienza dei richiedenti protezione internazionale. In Ambrosini M., Boccagni P., Piovesan S. (a cura di). L'immigrazione in Trentino. Trento: Cinformi.

Dovey K. (1985). Home and homelessness. In Altman I., Werner C. (eds.). Home Environments. New York: Plenum Press.

Duyvendak J.W. (2011). The politics of home. London: Palgrave.

Hall S. (2012). City, street and citizen: the measure of the ordinary. London: Routledge.

Jacobson K. (2009). A developed nature: a phenomenological account of the experience of home. Contemporary Philosophical Review, 42: 355-73. doi: 10.1007/s11007-009-9113-1.

Martini E.R., Torti A. (2013). Fare lavoro di comunità. Riferimenti teorici e strumenti operativi. Roma: Carocci Faber.

Marzorati R., Semprebon M. (2015). Building community in and out Via Padova 36. Sociologia urbana e rurale, 108: 69-85. doi: 10.3280/SUR2015-108005.

Miranda A. (2018). Ecuavolley in Madrid: new neighbours, old neighbours and the ordinary uses of home in public space. Fieldwork note. ERC HOMInG. Testo disponibile al sito: homing.soc.unitn.it.

Osti G., Ventura F. (a cura di). (2012). Vivere da stranieri in aree fragili. Napoli: Liguori.

Ripamonti E. (2003). L'arte di costruire una coalizione locale (inserto). Animazione sociale, 177: 46-56.

Ripamonti E. (2006). Sviluppo di comunità e progettazione partecipata. Skill, 31.

Rotter R. (2016). Waiting in the asylum determination process: Just an empty interlude? Time and Society, 25(1): 80-101. doi: 10.1177/0961463X15613654.

Semprebon M., Bonizzoni P., Marzorati R. (2017). L'immigrazione nei piccoli comuni italiani: processi, politiche, sfide. Mondi migranti, 1: 31-37. doi: 10.3280/MM2017001002.

Sigona N., Berg M., Gidley B. (eds.). (2014). Ethnography, diversity and urban space. London: Routledge.

Walters W. (2004). Secure borders, safe haven, domopolitics. Citizenship Studies, 8: 237260. doi: 10.1080/1362102042000256989.

Wessendorf S. (2010). Commonplace diversity: social relations in a superdiverse context. London: Palgrave.

Zapata R., Caponio T., Scholten P. (2017). Theorizing the "local turn" in a multigovernance framework of analysis. International Review of Administrative Sciences, 83(2): 241-246. doi: 10.1177/0020852316688426. 